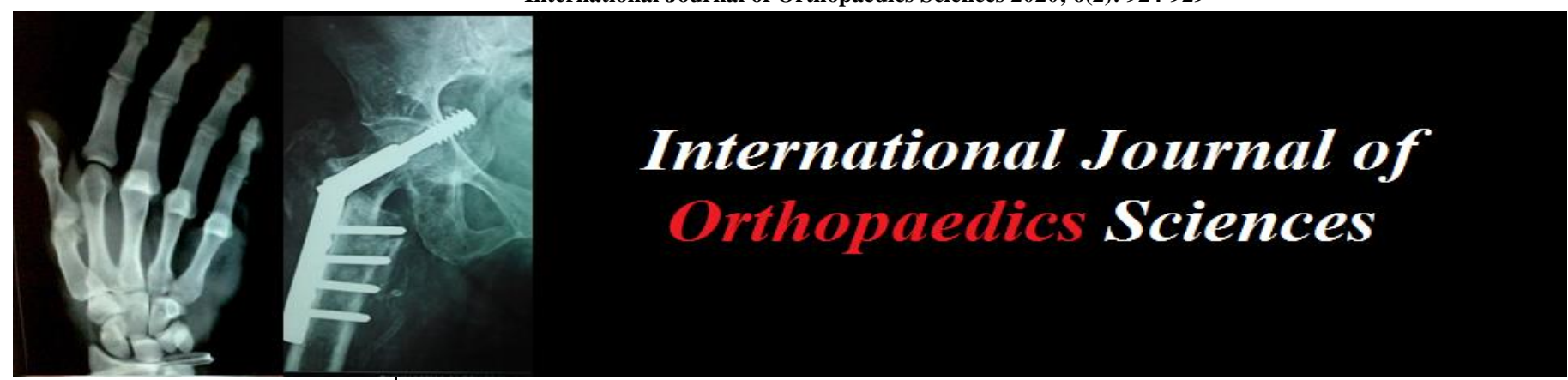

E-ISSN: 2395-1958

P-ISSN: 2706-6630

IJOS 2020; 6(2): 924-929

(C) 2020 IJOS

www.orthopaper.com

Received: 19-01-2020

Accepted: 20-02-2020

Dr. Vikram Sharma

Associate Professor, Department

of Orthopaedics, KD Medical

College and Hospitals, Mathura,

Uttar Pradesh, India

Dr. Vivek Chandak

Senior Resident, Department of Orthopaedics, KD Medical

College and Hospitals, Mathura,

Uttar Pradesh, India
Corresponding Author: Dr. Vikram Sharma Associate Professor, Department of Orthopaedics, KD Medical College and Hospitals, Mathura, Uttar Pradesh, India

\section{Comparative study of functional outcome in patients of Achilles tendinopathy treated with eccentric exercises and shockwave therapy}

\section{Dr. Vikram Sharma and Dr. Vivek Chandak}

DOI: https://doi.org/10.22271/ortho.2020.v6.i20.2162

\begin{abstract}
Achilles tendinopathy is a common clinical entity for which various modalities have been tried with variable results. This study was undertaken to compare the functional outcomes in patients of Achilles tendinopathy treated with eccentric exercises and shockwave therapy. 76 patients were included in the study and were randomly divided in two groups. Patients in Group 1 were treated with eccentric exercises and in Group 2 were treated with shock wave therapy.6 patients from Group 1 and 2 patients from Group 2 did not complete the duration of follow up and were excluded from the final analysis. Functional outcomes were evaluated using VAS scores and R-FFI scores at the start of intervention and at 6,12 and 24 weeks. Patients in both groups showed significant improvement $(p<0.01)$ across both the measures of outcome at each follow up visit. This indicated that both these modalities are effective in the treatment of Achilles tendinopathy. The difference in results was also significant $(p<0.01)$ when patients in Group 1 were compared with those in Group 2 indicating that eccentric exercises delivered better functional outcomes than shockwave therapy.
\end{abstract}

Keywords: Achilles tendinopathy, eccentric exercises, shock wave therapy

\section{Introduction}

Achilles tendinopathy is a difficult clinical problem. It occurs as an overuse injury in young active people, particularly runners and walkers ${ }^{[1,2]}$. Essentially it is a failed healing response to injury with haphazard proliferation of tenocytes, some evidence of degeneration in tendon cells, disruption of collagen fibres and subsequent increase in non-collagenous matrix ${ }^{[3]}$.

Various modalities have been proposed for the treatment of this condition like rest, strength and flexibilityexercises, anti-inflammatory agents, corticosteroids, adjuvant use of night splints ${ }^{[4]}$, adjuvant use of air heel brace ${ }^{[5,6]}$, therapeutic ultrasound ${ }^{[7]}$, shockwave therapy ${ }^{[8]}$ and prolotherapy ${ }^{[9]}$. However the results have not been conclusive. A Cochrane review showed that there was insufficient evidence from the randomized clinical trials to determine which of more than 20 methods was the most appropriate to manage this condition ${ }^{[10]}$.

Stanish et al. ${ }^{[11,12]}$ described the concept of eccentric exercises as treatment for tendinopathy and is based on the belief that tendon injuries often occur during the eccentric phase of muscle work and eccentric loading exercises probably help by promoting collagen fiber cross-linkage formation within the tendon. Various studies have demonstrated the effectiveness of the eccentric exercise regime ${ }^{[13-19]}$.

The rationale, proposed to use low energy shockwave treatment, for addressing, the failed healing response is that it stimulates soft-tissue healing, enhances angiogenesis, and inhibits pain receptors $[20,21,22]$. Pilot studies investigating the effects of shock wave therapy on Achilles tendinopathy have been promising [23, 24, 25], and randomized controlled trials, which used repetitive shock wave treatment without local anesthesia at weekly intervals with a primary follow-up of at least twelve weeks after treatment, found that shock wave therapy in the treatment of midsubstance Achilles tendinopathy was substantially more effective than

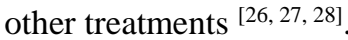

This study was designed to compare the efficacy of these two modalities in treating patients of Achilles tendinopathy. 


\section{Material and methods}

This prospective study was carried out at KD Medical College, Mathura, a 500 bedded hospital with teaching facilities. Patients who presented in the Outpatients Department between January 2018 and July 2019 with heel pain and in whom Achilles tendinopathy was diagnosed based on a clinical assessment and musculoskeletal ultrasound using the following criteria were included in this study.

- Insidious onset of pain in the region of the Achilles tendon, aggravated by weight bearing activity;

- Pain located 2 to $6 \mathrm{~cm}$ proximal to the Achilles tendon insertion upon palpation.

- Gray-scale musculoskeletal ultrasound of the Achilles tendon showing diffuse or local thickening with or without irregular fibre orientation and hypoechoic areas within the mid-portion of the Achilles tendon

The patients had to meet the following additional criterion to be included in this study.

1. Aged 18 years or greater;

2. Achilles tendon symptoms (pain) present for a minimum of two months;

3. Report having pain rated at least 3 out of 10 on a VAS of score of $1-10$

4. Be able to walk household distances (more than $50 \mathrm{~m}$ ) without the aid of a walker, crutches or cane;

5. Be willing to attempt to not use additional treatments (such as shoe modifications, physiotherapy, foot orthoses/bracing, injections, or surgery) for the Achilles pain during the trial (12 weeks);

Patients who had any of the following conditions were excluded from this study

1. Pregnancy

2. Previous Achilles tendon surgery in the symptomatic lower limb

3. Previous Achilles tendon rupture in the symptomatic lower limb

4. Inflammatory arthritis (e.g. ankylosing spondylitis);

5. Metabolic or endocrine disorders (e.g. type I or II diabetes);

6. Treatment with heel lifts or calf muscle eccentric exercise within the previous three months;

7. Injection of local anaesthetic, corticosteroid or other pharmaceutical agent into the Achilles tendon or surrounding area within the previous three months;

Patients were told to stop all anti inflammatory medications and report 7 days later when baseline VAS and FFI-R scores were recorded and patients were randomly allotted one of the following groups

Group A - Treated by eccentric exercise training

Group B - Treated by shockwave therapy

Group A patients were then introduced into the calf muscle eccentric exercise program based on the Alfredson [14] method, but with gradual introduction of the exercises. In the beginning, the loading consisted of the body weight. The patients were standing with all their body weight on their affected leg. From an upright body position and standing with all body weight on the forefoot, with the ankle joint in plantarflexion, the calf muscle was loaded by having the patient lower the affected limb down by dorsiflexing the ankle until the heel was well below the level of the step with the ankle in maximum dorsiflexion. The exercises were performed with the knee straight to eccentrically load the gastrocnemius and with the knee flexed to eccentrically load the soleus. Patients only loaded the calf muscle eccentrically; no concentric loading was performed, as the patients were instructed to use the unaffected leg and/or their arms to get back to the start position. Patients aimed to complete 3 sets of 15 repetitions with 1 minute of rest between the sets twice a day 7 days per week for 12 weeks. Patients started with one set of 10 repetitions on the first day of exercises and gradually progressed to 3 sets of 15 repetitions by the seventh day, aiming to complete 3 sets of 15 repetitions twice a day by the second week of treatment. They were advised to continue the exercises through mild or moderate pain, stopping only if the pain became unbearable.

When these exercises could be completed with no pain or discomfort, the patients progressed to carry a rucksack containing $5 \mathrm{~kg}$ of books. They were advised to continue to add weight in multiples of $5 \mathrm{~kg}$ if they did not experience pain in the Achilles tendon by the end of the third set of the eccentric exercises. Patients were asked to refrain from other forms of physical therapy intervention. They were informed that calf muscle soreness and increased pain in the Achilles insertion could appear during the first 2 weeks of eccentric training. At 6 weeks, the patients were told to slowly return to their previous activity.

Patients were also advised to apply ice over and around the Achilles tendon for $15 \mathrm{~min}$ following completion of each exercise session. They were allowed to undertake their usual activities (rather than complete rest) after receiving the intervention, provided the amount of pain they experience in the Achilles tendon pain does not exceed level 5 on a $0-10$ pain scale, where 0 is no pain and 10 is worst pain imaginable during exercise/activity.

Patients in Group 2 were treated with shockwave therapy. Three sessions of 2000 pulses at a frequency of eight pulses per second were given at weekly intervals according to the recommendation by Rompe et al. ${ }^{[18]}$ The area of tenderness was treated in a circumferential pattern starting from the area of maximal tenderness. No local anaesthesis was used.

All patients were followed up and assessed at 6,12 and 24 weeks after starting treatment. For VAS scores and Revised Foot Function Index ${ }^{[29]}$ score, which has been noted to be a well-developed measure of foot health related quality of life. [30]. The FFI-RS questionnaire consists of 34 questions grouped into 5 subcategories: pain (7 questions), stiffness (7 questions), difficulty (11 questions), activity limitation (3 questions), and social issues (6 questions). The answers are represented by a 4-level Likert scale. The numerical 5 is not a Likert scale; it is an option that the particular question is not applicable. The scoring of the FFI-R is based on the assumption that the severity of impairment in foot function is determined by the combination of pain, stiffness, difficulty, activity limitation, and psychosocial scales. A score is derived for each item by marking the patient experience on the choices displayed on a Likert scale. For example, on the pain subscale, grade 1 corresponds to no pain and 4 corresponds to worst pain imaginable. The numerical 5 is not used as a score but is used to indicate that the subscale question is not applicable for the patient. To obtain a subscale score, the item scores for a subscale are added and divided by the maximum total possible for the subscale items that the patient indicated were applicable, after which they are multiplied by 100.This method of scoring was applied to all subscales. The FFI-R short form score was the average of the five subscale scores. The significance level was established at a $p$ value of less than 0.05 . 
Compliance was assessed by direct questioning as to whether they completed the exercise regime as instructed.

\section{Results}

76 patients satisfied our criterion and were included in the study. 38 patients were randomly allocated to each group.

Of these $68(88.1 \%)$ patients completed the entire 12-week program. $8(11.9 \%)$ patients did not complete the treatment due to perceived failure. Of these 6 patients were from Group 1and 2 were from Group 2. These 8 patients were excluded from the final analysis. A total of 68 patients, (32 patients from Group 1 and 36 patients from Group 2) were available for final analysis. The mean age of patients in group 1 was 42.4 years, while those in Group 2 was 43.6 years. In Group 1 there were 18 males and 14 females whereas in group 2 there were 20 males and 16 females. Mean duration of symptoms in Group 1 was 17 weeks and in Group 2 was 19 weeks.
Table 1: Demographic data on 68 patients available for final analysis

\begin{tabular}{|c|c|c|}
\hline & Group 1 & Group 2 \\
\hline Mean Age (yrs) & 42.4 & 43.6 \\
\hline Sex- M/F & $18 / 14$ & $20 / 16$ \\
\hline Mean BMI (kg/m2) & 26.2 & 25.8 \\
\hline Mean Duration of symptoms (in weeks) & 17 & 19 \\
\hline
\end{tabular}

The mean VAS in Group 1 prior to treatment was 7.3. By 6 weeks post-treatment, this had reduced to 2.8 ( $p<0.01$ on original VAS score), and 12 weeks post treatment to 1.8 ( $p<$ 0.01 on original VAS score) and 24 weeks post treatment it was 1.1. ( $p<0.01$ on original VAS score).

The mean VAS in Group 2 prior to treatment was 7.5. By 6 weeks post-treatment, this had reduced t0 3.4 ( $p<0.01$ on original VAS score), at 12 weeks post treatment to 2.7 ( $p<$ 0.01 on original VAS score) and 24 weeks postoperatively it was 2.3 ( $p<0.01$ on original VAS score)

Table 2: Mean VAS scores over period of time

\begin{tabular}{|c|c|c|c|c|}
\hline & Baseline & 6 Weeks & 12 Weeks & 24 Weeks \\
\hline Group 1 & 7.3 & $2.9(60.2 \%)$ & $1.8(75.3 \%)$ & $1.1(84.9 \%)$ \\
\hline Group 2 & 7.5 & $3.4(54.6 \%)$ & $2.79(64 \%)$ & $2.3(69.3 \%)$ \\
\hline
\end{tabular}

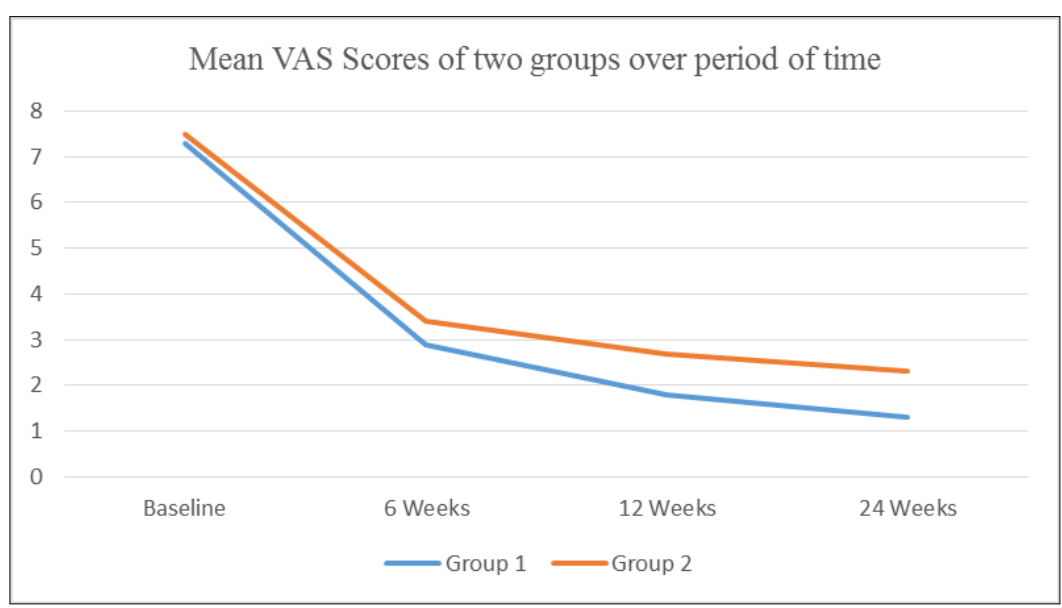

Fig 1: Mean VAS Scores of two groups over period of time

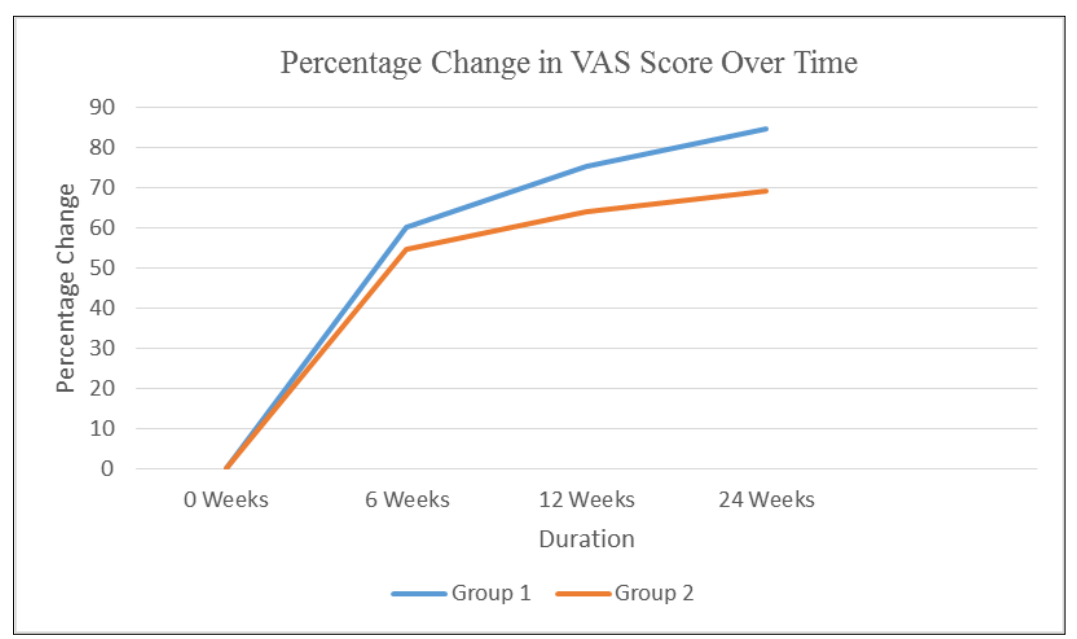

Fig 2: Percentage Change in VAS Score over Time

The mean R FFI score in Group A prior to treatment was 72.8. By 6 weeks post-treatment, this had reduced to 43.8 ( $p$ $<0.01$ on original VAS score), at 3 months post treatment to 38.7 ( $p<0.01$ on original VAS score) and 6 months post treatment it was 36.6 ( $p<0.01$ on original VAS score). The mean VAS in Group B prior to treatment was 71.6. By 6 weeks post-treatment, this had reduced t0 $52.8(p<0.01$ on original VAS score), and 3 months post treatment to $46.4(p<$ 0.01 on original VAS score) and 6 months post intervention it was 42.8 ( $p<0.01$ on original VAS score). 
Table 3: Mean R FFI scores over period of time

\begin{tabular}{|c|c|c|c|c|}
\hline & Baseline & 6 Weeks & 12 Weeks & 24 Weeks \\
\hline Group 1 & 72.8 & $43.8(39.7 \%)$ & $38.7(46.8 \%)$ & $36.6(49.7 \%)$ \\
\hline Group 2 & 71.6 & $52.8(23.7 \%)$ & $46.4(32.1 \%)$ & $42.8(37.4 \%)$ \\
\hline
\end{tabular}

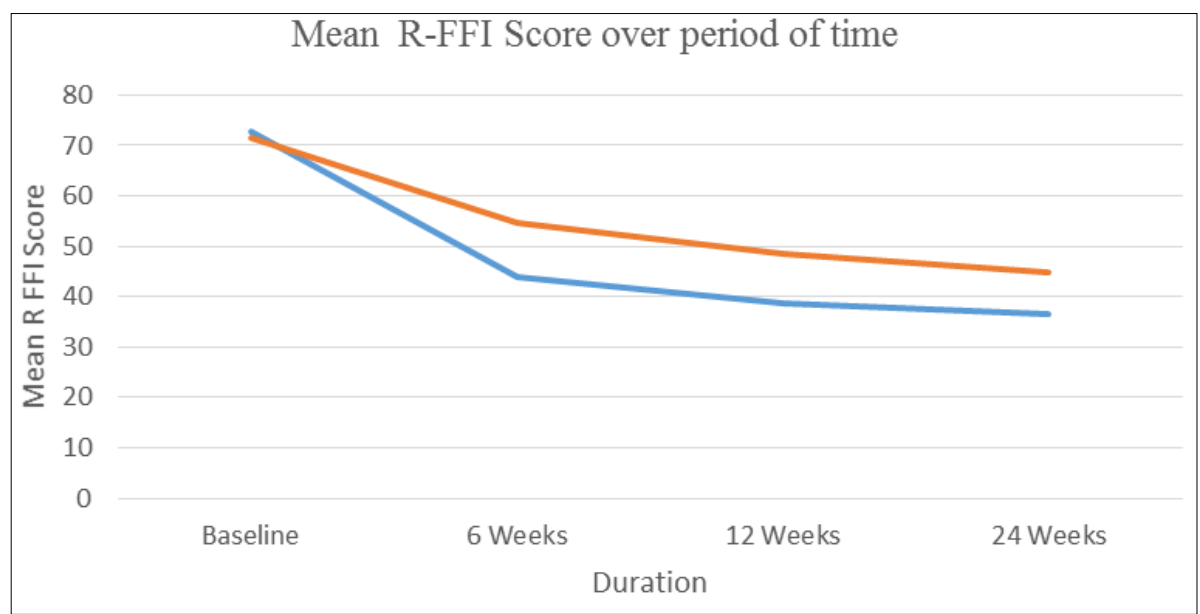

Fig 3: Mean R-FFI Score over period of time

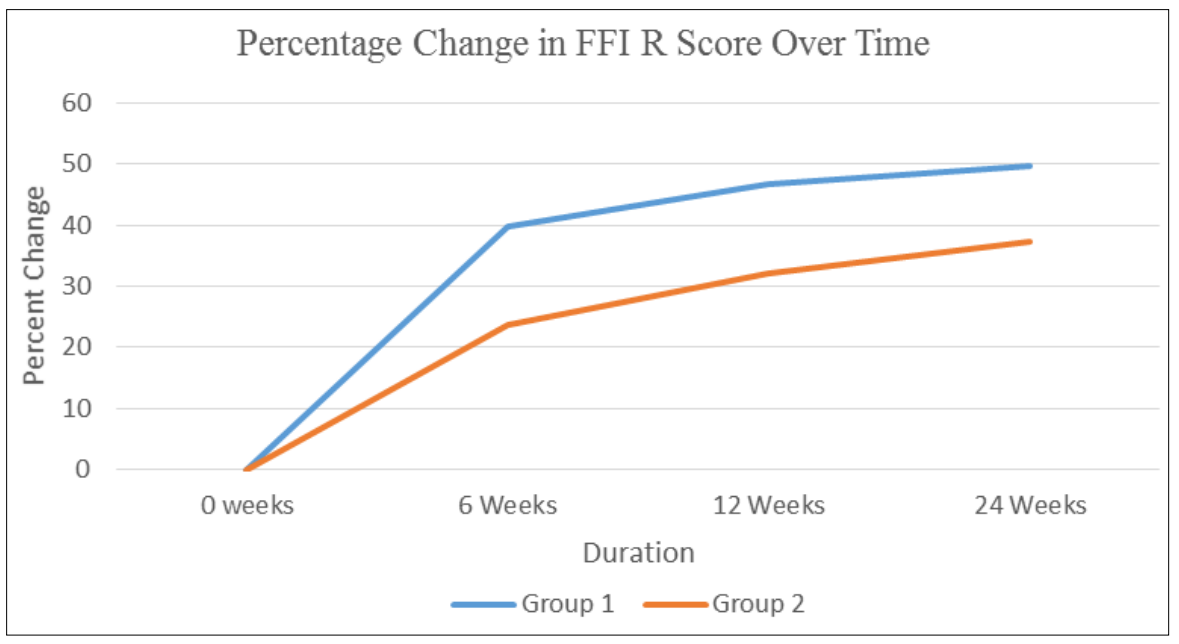

Fig 4: Percentage Change in R-FFI Score over Time

\section{Discussion}

Achilles tendinopathy is a very common clinical entity, presenting mostly in young, active people and causes significant morbidity. Various modalities have been tried to alleviate pain and improve function in these patients, but have met with variable results. In case of failure of non operative treatments, surgery is advised, but there has been no data from prospective, randomized, controlled trials ${ }^{[32-35]}$ and our understanding about surgery in this condition is based on descriptive studies. Eccentric training of calf muscles has been developed as an alternative to surgery and studies have demonstrated that painful eccentric calf- muscle training can provide excellent short-term results in up to $89 \%$ of patients with chronic painful midsubstance Achilles tendinopathy [1416, 36] Results of our study are in line with these studies demonstrating effectiveness of eccentric exercises. In our study at 6 weeks patients in Group 1 group showed significantly better results than baseline values. VAS score in Group 1 improved from 7.3 to 2.9 ( $\mathrm{p}$ value $<0.01$ ). whereas $\mathrm{R}$ FFI score improved from 72.8 to 43.8 (p value $<0.01$ ). Similar trend was seen when improvement in VAS and R-FFI scores were compared at 12 and 24 weeks. However, the good results of eccentric exercises have been challenged by certain investigators like Sayana et al. ${ }^{[19]}$, Woodley et al. ${ }^{[37]}$ and

\section{Kingma et al. ${ }^{[38]}$}

Low energy shockwave therapy has also been demonstrated to have beneficial effects in this condition. Studies by Astore et al. ${ }^{[23]}$, Perlick et al. ${ }^{[24]}$ and Peers et al. ${ }^{[25]}$, found that shock wave therapy in the treatment of midsubstance Achilles tendinopathy was substantially more effective than other treatments. The results of our study were similar to these studies. In our study at 6 weeks patients in Group 2 group showed significantly better results than baseline values. VAS score in Group 1 improved from7.5 to 3.4 ( $\mathrm{p}$ value<.01). whereas R FFI score improved from 71.6 to 52.8 (p value $<0.01)$. Similar trend was seen when improvement in VAS and R-FFI scores were compared at 12 and 24 weeks. However Costa et al. ${ }^{[26]}$ in a randomized, placebo-controlled trial in forty-nine patients with Achilles tendinopathy, found no difference in pain relief between the shock wave therapy group and the control group.

The limitation of our study was the small number of patients in each group. We had followed strict inclusion criterion. This contributed to minimizing the effects of confounding variables but also led to the decrease in the number of patients. Absence of blinding was another limitation of this study.

In our study the difference was found to be significant ( $p$ 
value $<0.01$ ) when results between Group 1 and Group 2 were compared at every follow up visit, indicating that eccentric loading was better than shockwave therapy in the management of these patients whereas Rompe et al. ${ }^{[20]}$, in a study of seventy-five patients with a chronic recalcitrant tendinopathy of the midsubstance of the Achilles tendon found that eccentric loading and shock wave therapy did not differ significantly, in functional outcomes but they showed substantially better results than the wait-and-see policy. Rompe et al. ${ }^{[31]}$ in another study found that the combined approach of painful eccentric loading plus repetitive lowenergy SWT produced significantly better results than eccentric calf muscle training alone. In our study we did not study the effects of combined therapy. Another finding was that in both the groups, improvement seen in the first 3 months was more than compared to the period between 3- 6 months, but in both the groups, results at 6 months were better than at 3 months. We could not find any explanation for this observation.

\section{Conclusion}

Based on the above findings we concluded that both eccentric exercises and shock wave therapy are effective in the treatment of Achilles tendinopathy with eccentric exercises showing better results than shockwave therapy in terms of functional improvement. However larger multicenter studies are required to further establish their efficacy.

\section{References}

1. Astrom M, Rausing A. Chronic Achilles tendinopathy: a survey of surgical and histopathological findings. Clin Ortho Related Res. 1995; 316:151-164.

2. Clement DB, Taunton JE, Smart GW. Achilles tendonitis and peritendonitis: etiology and treatment. Am J Sports Med. 1984; 12:179-184.

3. Maffulli N, Longo UG. Conservative management for tendinopathy: Is there enough scientific evidence? Rheumatology (Oxford). 2008; 47:390-391.

4. De Jonge $\mathrm{S}$, de Vos RJ, van Schie HT et al. One-year follow- up of a randomised controlled trial on added splinting to eccentric exercises in chronic midportion Achilles tendinopathy. Br J Sports Med. 2010; 44:673677. http://dx.doi.org/10.1136/bjsm.2008.052142

5. Knobloch K, Schreibmueller L, Longo UG, Vogt PM. Eccentric exercises for the management of tendinopathy of the main body of the Achilles tendon with or without the AirHeel Brace. A randomized controlled trial. A: effects on pain and microcirculation. Disabil Rehabil. 2008; 30:1685-91.

6. Petersen W, Welp R, Rosenbaum D. Chronic Achilles tendinopathy: a prospective randomized study comparing the therapeutic effect of eccentric training, the AirHeel brace, and a combination of both. The Am J Sports Med, 2007; 35(10):1659-67.

7. Chester R, Costa ML, Shepstone L et al. Eccentric calf muscle training compared with therapeutic ultrasound for chronic Achilles tendon pain - a pilot study. Man Ther. 2008; 13:484-91. http://dx. doi.org/10.1016/j.math.2007.05.014

8. Rompe JD, Furia J, Maffulli N. Eccentric loading compared with shockwave treatmentfor chronic insertional Achilles tendinopathy. A randomized controlled trial. J Bone Joint Surg Am. 2008; 90:52-61.

9. Yelland MJ, Sweeting KR, Lyftogt JA et al. Prolotherapy injections and eccentric loading exercises for painful
Achilles tendinosis: a randomised trial. Br J Sports Med. Jul 6. [Epub ahead of print], 2010.

10. Mc Lauchlan GJ, Handoll HH. Interventions for treating acute and chronic Achilles Tendinitis. Cochrane Database Syst Rev. 2001; 2:CD000232.

11. Stanish WD, Curwin S, Mandel S. Tendinitis: Its Etiology and Treatment. Oxford: Oxford University Press, 2000.

12. Stanish WD, Rubinovich RM, Curwin S. Eccentric exercise in chronic tendinitis. Clin Orthop, 1986, 65-68.

13. Alfredson $\mathrm{H}$, Cook J. A treatment algorithm for managing Achilles tendinopathy: new treatment options. Br J Sports Med. 2007; 41:211-216.

14. Alfredson H, Pietila T, Jonsson P, Lorentzon R. Heavyload eccentric calf muscle training for the treatment of chronic Achilles tendinosis. Am J Sports Med. 1998; 26:360-366.

15. Fahlstrom M, Jonsson $\mathrm{P}$, Lorentzon R, Alfredson $\mathrm{H}$. Chronic Achilles tendon pain treated with eccentric calfmuscle training. Knee Surg Sports Traumatol Arthrosc. 2003; 11:327-333.

16. Ohberg L, Lorentzon R, Alfredson H. Eccentric training in patients with chronic Achilles tendinosis: normalised tendon structure and decreased thickness at follow up. $\mathrm{Br}$ J Sports Med. 2004; 38:8-11.

17. Maffulli N, Walley G, Sayana M, Longo UG, Denaro V. Eccentric calf muscle training in athletic patients with Achilles tendinopathy. Disabil Rehabil. 2008, 10. [Epub ahead of print]

18. Rompe JD, Nafe B, Furia JP, Maffulli N. Eccentric loading, shock- wave treatment, or a wait-and-see policy for tendinopathy of the main body of tendo Achillis: a randomized controlled trial. Am J Sports Med. 2007; 35:374-383.

19. Sayana MK, Maffulli N. Eccentric calf muscle training in non-athletic patients with Achilles tendinopathy. J Sci Med Sport. 2007; 10:52-58.

20. Furia JP. High-energy extracorporeal shock wave therapy as a treatment for insertional Achilles tendinopathy. Am J Sports Med. 2006; 34:733-40.

21. Wang CJ, Wang FS, Yang KD, Weng LH, Sun YC, Yang YJ. The effect of shock wave treatment at the tendonbone interface - an histomorphological and biomechanical study in rabbits. J Orthop Res. 2005; 23:274-80.

22. Lohrer H, Scholl J, Arentz S. [Achilles tendinopathy and patellar tendinopathy. Results of radial shockwave therapy in patients with unsuccessfully treated tendinoses]. Sportverl Sportschad. 2002; 16:108-14. German.

23. Astore F, Sansone V, De Marchi F. Extracorporeal shock wave therapy for tendinopathy of Achilles. Read at the 23rd Isokinetic Congress on the Rehabilita- tion of Sports Muscle and Tendon Injuries; Turin, Italy, 2004.

24. Perlick L, Schiffmann R, Kraft CN, Wallny T, Diederich O. [Extracorporeal shock wave treatment of the achilles tendinitis: Experimental and preliminary clinical results]. Z Orthop Ihre Grenzgeb. German, 2002; 140:275-80.

25. Peers K. Extracorporeal shock wave therapy in chronic achilles and patellar tendinopathy. Leuven, Belgium: Leuven University Press, 2003.

26. Costa M, Shepstone L, Donell S, Thomas T. Shock wave therapy for chronic Achilles tendon pain: a randomized placebo-controlled trial. Clin Orthop Relat Res. 2005; 440:199-204.

27. Chen YJ, Wang CJ, Yang KD, Kuo YR, Huang HC, 
Huang YT, Sun YC et al. Extracorporeal shock waves promote healing of collagenase-induced Achilles tendinitis and increase TGF-beta1 and IGF-I expression. J Orthop Res. 2004; 22:854-61.

28. Rompe JD, Furia J, Maffulli N. Eccentric loading compared with shock wave treatment for chronic insertional achilles tendinopathy: a randomized, controlled trial. J Bone Joint Surg Am. 2008; 90:52-61.

29. Budiman-Mac E, Conrad K, Stuck R, Matters M. Theorotical model and Rasch analysis to develop a revised a Foot Function Index. Foot Ankle Int. 2006; 27:519-27.

30. Walmsley S, Williams A, Ravey M, Graham A. The rheumatoid foot: a systemic literature review of patient reported outcome measures. J Foot Ankle Res, 2001; $3: 12$.

31. Rompe JD, Furia JP, Maffulli N. Eccentric Loading Versus Eccentric Loading plus Shock-Wave Treatment for Midportion Achilles Tendinopathy. A Randomized Controlled Trial Am J Sports Med. 2009; 37:463-470.

32. Maffulli N, Sharma P, Luscombe KL. Achilles tendinopathy: aetiology and management. J R Soc Med. 2004; 97:472-6.

33. Krishna Sayana M, Maffulli N. Insertional Achilles tendinopathy. Foot Ankle Clin. 2005; 10:309-20.

34. Wagner E, Gould JS, Kneidel M, Fleisig GS, Fowler R. Technique and results of Achilles tendon detachment and reconstruction for insertional Achilles tendinosis. Foot Ankle Int. 2006; 27:677-84.

35. Maquirriain J. Endoscopic Achilles tenodesis: a surgical alternative for chronic insertional tendinopathy. Knee Surg Sports Traumatol Arthrosc. 2007; 15:940-3.

36. Roos EM, Engstrom M, Lagerquist A, Soderberg B. Clinical improvement after 6 weeks of eccentric exercise in patients with mid-portion Achilles tendinopathy-a randomized trial with 1-year follow-up. Scand J Med Sci Sports. 2004; 14:288-95.

37. Woodley BL, Newsham-West RJ, Baxter GD. Chronic tendinopathy: effective- ness of eccentric exercise. Br J Sports Med. 2007; 41:188-99.

38. Kingma JJ, de Knikker R, Wittink HW, Takken T. Eccentric overload training in patients with a chronic Achilles tendinopathy: a systematic review. Br J Sports Med. 2007; 41:e3. 